Nonlinear Processes in Geophysics (2005) 12: 157-162

SRef-ID: $1607-7946 / \mathrm{npg} / 2005-12-157$

European Geosciences Union

(c) 2005 Author(s). This work is licensed

under a Creative Commons License.

\title{
Multifractal analysis for the ULF geomagnetic data during the 1993 Guam earthquake
}

\author{
Y. Ida ${ }^{1}$, M. Hayakawa ${ }^{1}$, A. Adalev ${ }^{1}$, and K. Gotoh ${ }^{2}$ \\ ${ }^{1}$ Department of Electronic Engineering, The University of Electro-Communications, 1-5-1 Chofugaoka, Chofu Tokyo \\ 182-8585, Japan \\ ${ }^{2}$ National Institute of Information and Communications Technology (NiCT), 3-4 Hikarino-oka, Yokosuka Kanagawa, \\ 239-0847, Japan
}

Received: 7 September 2004 - Revised: 9 December 2004 - Accepted: 3 January 2005 - Published: 31 January 2005

\begin{abstract}
In our previous papers we have shown that the fractal (monofractal) dimension $\left(D_{o}\right)$ showed a significant increase before the Guam earthquake occurred on 8 August, 1993. In order to have a further support to this precursory effect to the general rupture (earthquake) we have carried out the corresponding multifractal analysis (by means of detrended fluctuation analysis) for the same data to study the statistical self-similar properties in a wide range of scales. We have analyzed the ULF geomagnetic data (the most intense $H$ component) observed at Guam observatory. As the result, we have found that we could observe significant changes in the multifractal parameters at Guam such that $\alpha_{\min }$ showed a meaningful decrease about 25 days before the earthquake and correspondingly $\Delta \alpha$ increased because $\alpha_{\max }$ exhibited no significant change at all. The most sensitive parameter seems to be non-uniformity factor $\Delta$. Correspondingly, the generalized multifractal dimension $D q$ $(q>1)$ showed a significant decrease (whereas $D q(q<0)$ showed no change) and $D_{0}(=D q(q=0)$ (as already found in our previous papers) is reconfirmed to increase before the earthquake. These multifractal characteristics seem to be a further support that these changes are closely associated with the earthquake as a precursor to the Guam earthquake, providing us with appreciable information on the pre-rupture evolution of the earthquake.
\end{abstract}

\section{Introduction}

An important question in geophysics and in material science is to investigate whether any precursory signature exists or not before a macroscopic rupture or an earthquake and then to identify such an earthquake precursor if it exists.

We understand that when a heterogeneous material is strained, its evolution toward the final breaking is characterized by the nucleation and coalescence of microcracks before

Correspondence to: M. Hayakawa

(hayakawa@whister.ee.uec.ac.jp) the final rupture. The two physical quantities are recognized as being most indicative of microfracturing process in the focal zone; that is, (1) ULF electromagnetic emissions and (2) acoustic emissions (Hayakawa, 2001; Hayakawa et al., 2004). There have recently been found a lot of convincing evidences on the electromagnetic emissions in a wide frequency range from DC, ULF to VHF associated with earthquakes, which are known to take place prior to the final breakup (e.g. Hayakawa and Fujinawa, 1994; Hayakawa, 1999; Hayakawa and Molchanov, 2002). Our main tool in this paper is to monitor the microfractures which are known to occur before the final breakup in the focal zone of an earthquake, by recording the ULF emissions. The presence of precursory signature of earthquakes is clearly identified in the ULF range for large (magnitude greater than 7) earthquakes such as Spitak, Loma Prieta, Guam, Biak etc. (Fraser-Smith et al., 1990; Mochanov et al., 1992; Kopytenko et al., 1993; Hayakawa et al., 1996, 1999, 2000).

The ULF emissions are found to take place from a few weeks to a few days prior to large destructive earthquakes (including Spitak, Loma Prieta, Guam etc.), which are considered as the so-called precursors of the general fracture. The emissions in the higher frequency range (like, VLF and HF/VHF) (Kapiris et al., 2004) are likely to be occurring in the focal zone of an earthquake, but they cannot be detected on the Earth's surface because of their extremely severe attenuation in the crust. So, those higher frequency emissions might be generated as secondary effect near the Earth's surface, and their generation mechanism is very unclear at the moment. In comparision with this, the ULF emissions are very definite to have been generated in the focal zone and to have propagated up to the subsurface ULF sensors. We can list the following reasons for this.

(1) When we estimate the skin-depth for ULF waves (period: 0.1 to $200 \mathrm{~s}$ ) for the earthquake source depths from $5 \mathrm{~km}$ to $100 \mathrm{~km}$, it will be less than $100 \mathrm{~km}$. Hence, we can expect that those ULF waves are definitely coming from the source region. 


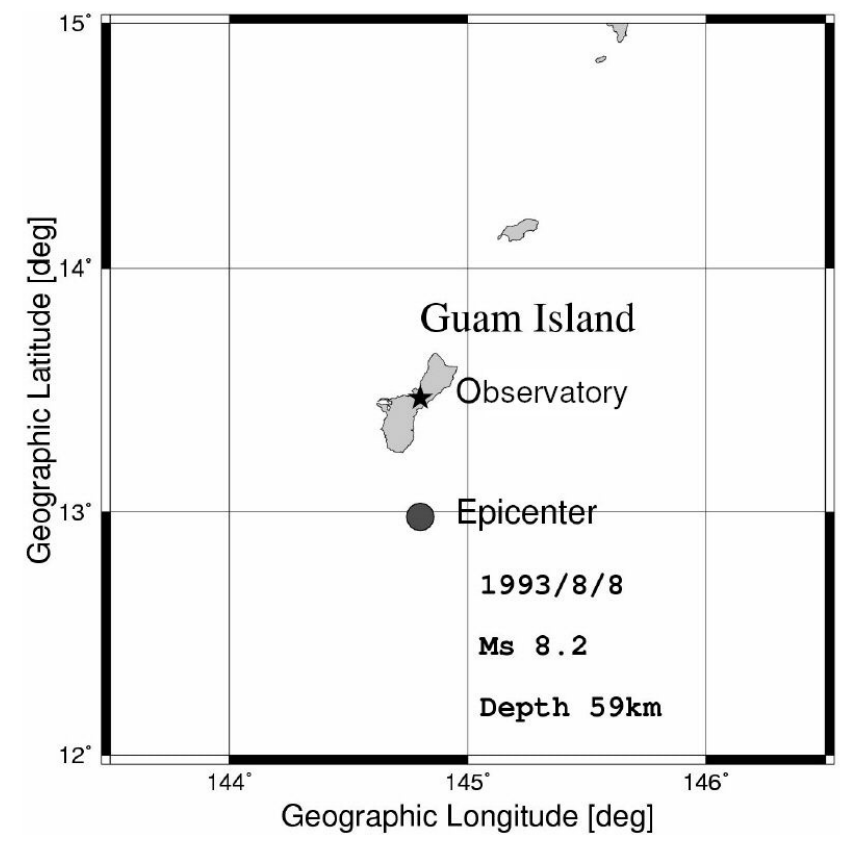

Fig. 1. Relative location of our ULF observing station and the epicenter of the earthquake, together with the earthquake characteristics.

(2) Dynamic process in seismo-active areas can produce current systems of different kinds (see e.g. Molchanov and Hayakawa, 1995; Vallianatos and Tzanis, 1999 and references therein), which can be local sources for electromagnetic waves at different frequencies. The ULF range is the most possible to come from the source region with the least attenuation.

Based on these arguments, we can consider that those ULF emissions would carry the information on the microfracturing taking place near the focal zone. For example, as already mentioned before, Molchanov and Hayakawa (1995) proposed the generation mechanism of seismogenic ULF emissions on the basis of microfracturing.

One more important notice for those ULF emissions is the necessity of sophisticated signal processing. Signal processing is found to be of potential significance in finding out weak seismogenic ULF emissions especially in the general case when the observatory is located relatively away from the epicenter, so that the seismogenic emission is embedded in the noise. We have proposed so far several possible methods, including the polarization analysis method (using the ratio of vertical to horizontal magnetic field components) (Hayakawa et al., 1996), fractal (mono-) analysis (Hayakawa et al., 1999), principal component analysis (PCA) (Gotoh et al., 2002) etc. The first and third methods are simply mathematical, but the second is a physical analysis method, which was exactly the first step of this paper.

Because the dynamics of earthquakes is well known to exhibit properties which are characteristics for the selforganized criticality (SOC) state (e.g. Bak et al., 1987; Bak, 1997), we have made the first attempt to use the fractal anal- ysis to the seismogenic ULF emissions for the Guam earthquake (Hayakawa et al., 1999). Since the principal feature of the SOC state is a fractal organization of the output parameters both in space (scale-invariant structure) and in time (flicker noise or 1/f noise). If the time series of ULF data is a temporal fractal, we expect a power-law spectral density of the recorded time series: $S(f) \propto f^{-\beta}$ ( $\beta$ : spectral exponent). Hayakawa et al. (1999) have found a significant change in this spectral exponent $(\beta)$ just before the Guam earthquake in such a way that the value of $\beta$ is approaching unity (becoming flicker noise) before the rupture, and later the similar behavior has also been confirmed for another large earthquake at Biak (Hayakawa et al., 2000). This estimation of fractal dimension was based on the spectral slope, $\beta$ in the spectral analysis, but later Smirnova et al. (2001) and Gotoh et al. $(2003,2004)$ have compared different analysis methods in estimating the fractal dimension (spectral slope, Burlaga and Klein (1986) and Higuchi (1988) methods). Their conclusion is that the Higuchi method provides us with the most reliable value of the fractal dimension. The seismicity spatial and temporal distribution is known to reveal statistically selfsimilar properties in a wide range of scales and can be tread as multifractal (e.g. Turcotte, 1997). Our previous studies are just a monofractal analysis, but we are interested in examining the multifractal nature of the ULF electromagnetic data for the same Guam earthquake.

This paper is a further extension of our previous papers dealt with the monofractal analysis (Hayakawa et al., 1996, 2000; Smirnova et al., 2001; Gotoh et al., 2003, 2004). We have already found a significant precursory change in the monofractal dimension before the Guam earthquake (Hayakawa et al., 1999; Smirnova et al., 2001; Gotoh et al., 2003, 2004). This data set is also studied in this paper, to which we have applied the multifractal analysis, and this will be the first attempt in seismogenic ULF data.

\section{Experimental ULF data and analysis procedure}

The details of the ULF data for the Guam earthquake have already been given in Hayakawa et al. (1999), but we have to repeat only the important points as follows. The Guam earthquake with magnitude $M s=8$, occurred on 8 August 1993 at 08:34 UT suddenly and without any foreshock. Its epicenter was located in the sea near the Guam island (geographic coordinates: $12.98^{\circ} \mathrm{N}, 1444.80^{\circ} \mathrm{E}$ ), and its depth was $60 \mathrm{~km}$. The Guam observatory where the ULF data were recorded, is located at $\sim 65 \mathrm{~km}$ from the epicenter. Figure 1 illustrates the relative location of our ULF observatory with respect to the epicenter. A regular magnetic observation is maintained there using a three-axis ring-core-type fluxgate magnetometer (Hayakawa et al., 1996). Three components of magnetic variations are usually recorded on a digital cassette tape with a sampling rate of $1 \mathrm{~s}$, but we use only the $H$ component (which is the most intense) (though Gotoh et al., 2004 have already found the similar behavior in the monofractal dimension for the $Z$ component as well). We analyze the data 
during one whole year of 1993, and we analyze the data during daytime (LT=14:00-15:00), because Gotoh et al. (2004) have found that the most significant change in the monofractal dimension was observed for the Guam earthquake during daytime. One hour data are treated, so that the number of data is 3600 points per day.

We now describe the multifractal analysis. The general description can be found in Feder (1988) and Mandelbrot (1989). There have been developed a few methods for multifractal analysis, but we have adopted the MFDFA (multifractal detrended fluctuation analysis) (Kanterhardt et al., 2002). The MFDFA is apparent to be much more accurate than the conventional box counting, and the detrended fluctuation analysis (DFA) has become a widely-used technique for the determination of fractal scaling properties and the detection of long-range correlations in noisy, nonstationary time series (Gotoh et al., 2004). One reason to employ the DFA method is to avoid spurious detection of correlations that are artifacts of nonstationariteis in the time series. The data used are not filtered by using any filter before the multifractal analysis. We followed the analysis of DFA method developed by Kantelhardt et al. (2002). Let us suppose that $x_{k}$ is a time series of length $N$ ( $N=3600$ points for one hour every day), and we first determine the profile

$Y(i) \equiv \sum_{k=1}^{i}\left[x_{k}-\langle x\rangle\right](i=1, \cdots, N)$

(where $\langle x\rangle$ is the mean over our relevant one-hour interval), which means that we remove the DC bias. Next we divide the profile $Y(i)$ into $N_{s} \equiv$ int $(\mathrm{N} / \mathrm{s})$ (int means integer) non-overlapping segments of equal length $s$. Since the length $N$ of the series is often not a multiple of the considered time scales, a short part at the end of the profile may remain. In order not to disregard this part of the series, the same procedure is repeated starting from the opposite end. Thereby, $2 N_{s}$ segments are obtained altogether. Then, we calculate the local trend for each of the $2 N_{s}$ segments by a least-square fit of the series and we determine the variance;

$F^{2}(v, s) \equiv \frac{1}{s} \sum_{i=1}^{s}\left\{Y[(v-1) s+i]-y_{v}(i)\right\}^{2}$

for each segment

$v(v=1, \cdots, N s)$

and

$F^{2}(v, s) \equiv \frac{1}{s} \sum_{i=1}^{s}\left\{Y[(v-1) s+i]-y_{v}(i)\right\}^{2}$

for $v=N_{s}+1, \cdots, 2 N_{s}$. Here, $y_{v}(i)$ is the fitting polynomial in segment $v$. We have used the sixth-order $(m=6)$ polynomials in the fitting procedure. In the paper by Kantelhardt et al. (2002) they have used the values of $m=2$ or 3 , but we could not get sufficient fitting for these $m$ values. Simply increasing $\mathrm{m}$ value, we have found that $m=6$ gave sufficient

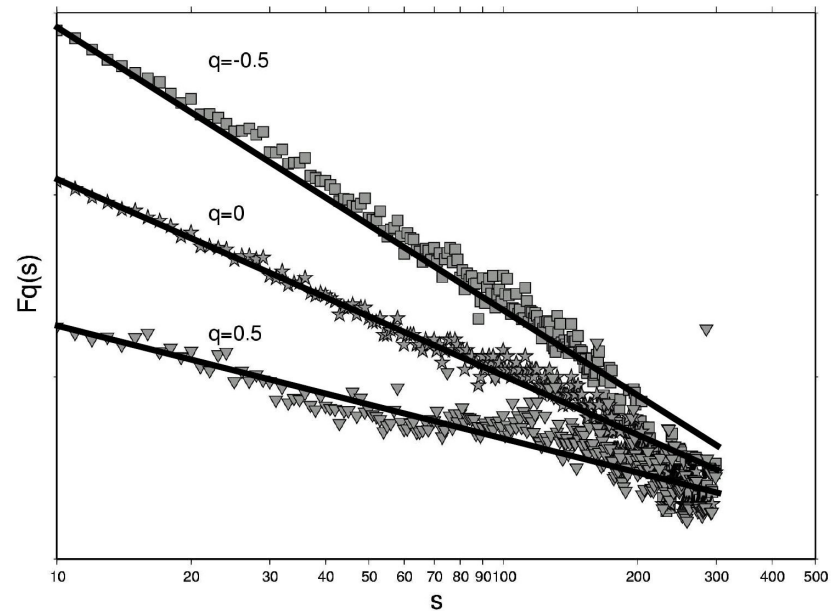

Fig. 2. Examples of $F q(s)-s$ curves on a particular day of 12 March 1993, for a few selected $q$ values $(q=-0.5,0,+0.5)$.

fitting. We then average over all segments to obtain the qth order fluctuation function.

$F q(s) \equiv\left\{\frac{1}{2 N_{s}} \sum_{\nu=1}^{2 N_{s}}\left[F^{2}(\nu, s)\right]^{q / 2}\right\}^{1 / q}$.

Of course, $F q(s)$ depends on the DFA order $m$, and by construction $F q(s)$ is only defined for $s \geq m+2$. Kantelhardt et al. (2002) have suggested the value for $s$ in such a way that $s<N / 4$. So that we have adopted a reasonable range of $s=10 \sim 300$, and so the obtained results are acceptable. We show one example of $F q(s)(q=-0.5,0,+0.5)$ on a particular day of 12 March 1993 in Fig. 2. This figure shows the s dependence of $F q$, which indicates that $F q(s)$ decreases as a power law for large values of $s$ if the series $x_{k}$ are longrange power-law corrected, and we can estimate the $h(q)$, the generalized Hurst exponent. It is seen that $F q(s)$ can be approximated as $\propto s^{\tau(q)}$, so that we can estimate the $\tau(q)$. Here we had better comment on how good the fitting of the fluctuation function $F q(s)$ by a linear approximation is. One way to estimate this is the use of $r 2$ values as the square of the correlation coefficient. The $r 2$ values are found to be over 0.8 in Fig. 2, which are not so bad as compared with the corresponding values on the monofractal estimation by Gotoh et al. (2004).

On the basis of obtained $\tau(q)$ characteristics, we can estimate the well-known curves of $f(\alpha)-\alpha$ (where $\alpha$ is the Lipschitz-Hölder exponent) and $D q$ curve by means of the following relationships $(\alpha(q)=d \tau(q) / d q$, $f(\alpha(q))=q \alpha(q)-\tau(q)$, and $D(q)=D q=\tau(q) /(q-1)$ ) (see the textbooks by Goltz (1997) and Feder (1988)). Figure 3 is an example ((a) $D(q)$ curve and (b) $f(\alpha)-\alpha$ curve) obtained for the same day on 12 March 1993. The multifractal parameters characterizing this $f(\alpha)$ spectrum are (1) $\alpha_{\min },(2) \alpha_{\max }\left(\alpha_{\min }\right.$ and $\alpha_{\max }$ satisfy $\left.f(\alpha)=0\right)$, (3) $w\left(\equiv \alpha_{\max }-\alpha_{\min }\right)$, (4) $f_{\max }$, (5) $\alpha\left(f_{\max }\right)$, and (6) nonuniformity factor $\Delta \equiv\left(\frac{\alpha_{\max }-\alpha_{\min }}{f_{\max }}=\frac{w}{f_{\max }}\right)$. Figure $3 \mathrm{~b}$ can 

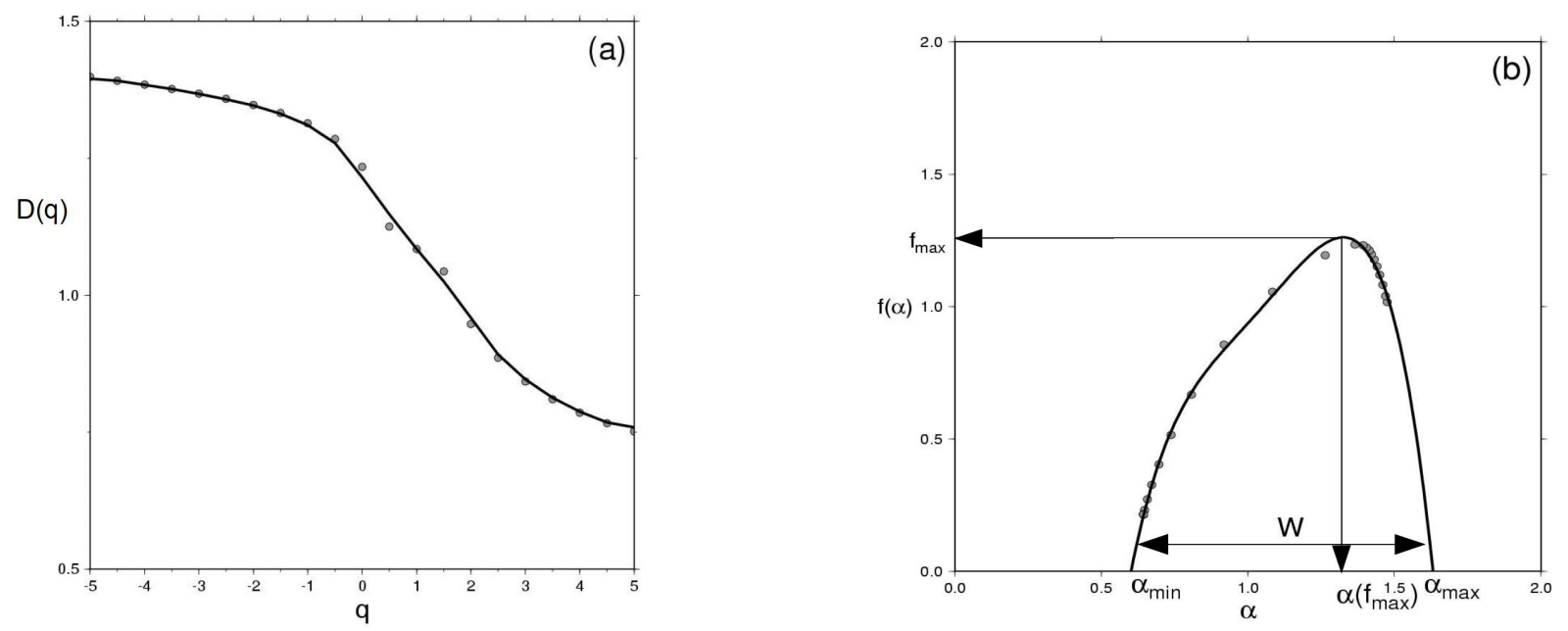

Fig. 3. An example of (a) $D q-q$ curve and (b) $f(\alpha)-\alpha$ curve on the same day of 12 March 1993 .

be interpreted as follows: The spectrum which looks like an upside-down parabola, peaks at $f_{\max }$ (at $\left.\alpha\left(f_{\max }\right)\right)$ and stretches from $\alpha_{\min }$ to $\alpha_{\max }$. That is, the range $w\left(\alpha_{\min }\right.$ to $\left.\alpha_{\max }\right)$ quantifies the non-uniformity of the fractal, while $f(\alpha)$ tells how frequently events with scaling exponent $\alpha$ occur. Figure 4 illustrates the whole-one year plot of the variations of those multifractal parameters. The thin lines are the daily variations (the corresponding value for one particular day), and the full lines indicate the running average over \pm 5 days on a current day ( +5 days including the current day and -5 days including the current day; Total 9 days). The vertical line in all the plots (as shown as 8/8) indicates the date of the Guam earthquake (i.e. 8 August). The top panel is the temporal evolution of geomagnetic activity expressed by the $A_{p}$ index. The multifractal parameters are given from top; $\Delta$, $\alpha\left(f_{\max }\right), f_{\max }, w, \alpha_{\max }$, and $\alpha_{\min }$. By means of a close look at Fig. 4, we can notice the following important findings for the multifractal parameters.

1. The temporal variations of bottom three panels $\left(\alpha_{\min }\right.$, $\alpha_{\max }$, and $w$ ) has indicated a significant change (increase) in $w$ about 30 days before the earthquake. This is entirely dependent on the significant decrease in $\alpha_{\min }$, because $\alpha_{\max }$ is not found to exhibit any obvious changes.

2. $\alpha\left(f_{\max }\right)$ seems to show no significant change, but $f_{\max }$ itself is found to indicate a significant decrease before the earthquake.

3. The most pronounced effect is clearly seen in the temporal evolution of the non-uniformity factor, $\Delta$. That is, the value $\Delta$ of is found to show a significant increase about 30 days before the earthquake and remain at a high value until a few days before the earthquake. This $\Delta$ value is seen to be back to the background value a few months later than the earthquake.
4. Significant changes (increase) in $\Delta$ before the earthquake are apparent to have no correlation with the geomagnetic activity because we are during geomagnetically quiet period.

In order to give some more quantitative estimate on the significant change in $\Delta$ (conclusions (3) and (4)), we have plotted the mean value (over the whole period) (horizontal line) and the corresponding $1 \sigma(\sigma$ : standard deviation (over the whole year)). Even the running mean (over 9 days) of $\Delta$ is found to exceed the mean plus one standard deviation, and the daily $\Delta$ is seen to fluctuate at a high level (like the mean plus $2 \sigma$ ). This behavior is apparent to be very abnormal.

Another form of presentation is the use of $D q(q)$ curve for the same one year of 1993, including the earthquake day of 8 August. The result is given in Fig. 5, in which the ordinate is the value of $q$ and the value of $D q$ is indicated in different color. We can notice the following facts.

(1) As for $D_{o}$ (capacity dimension) we find a significant increase about 30 days before the earthquake, which seems to support the previous works by Hayakawa et al. (1990) and Gotoh et al. (2004) based on the monofractal analysis. The most significant effect is seen for $q=0.5$.

(2) In the region of $q>1, D q$ is found to decrease about 30 days before the earthquake, while $D q(q<0)$ did not show any particular changes.

\section{Discussion and conclusion}

In our previous monofractal analyses by Hayakawa et al. (1999) and Gotoh et al. (2004), we have already found that the monofractal dimension (corresponding to $D_{o}$ in this analysis) showed a significant increase before the Guam earthquake, which seems to be a precursor (Hayakawa et al., 1999) to the Guam earthquake (Hayakawa et al., 1999). In addition to this monofractal analysis, our first attempt of multifractal analysis (by means of MFDFA) in this paper 


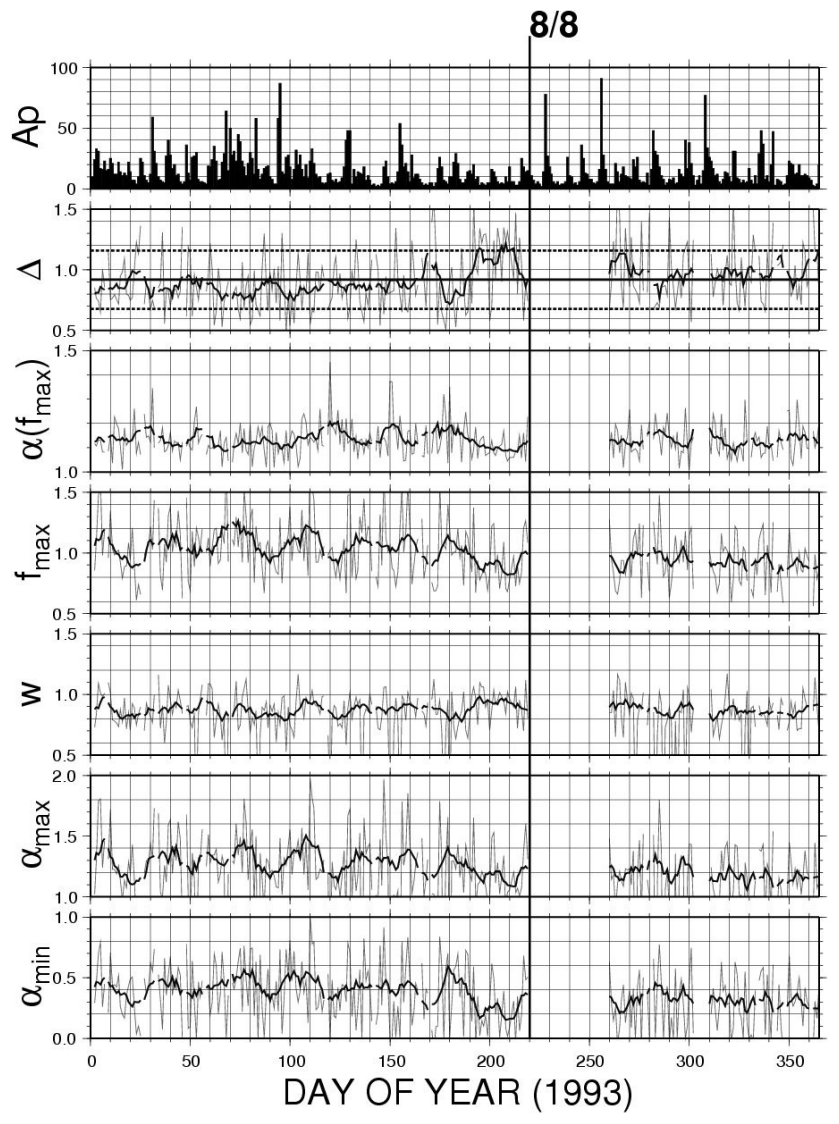

Fig. 4. Temporal evolution of multifractal parameters during a whole year of 1993 . From the bottom, $\alpha_{\min }, \alpha_{\max }, w, f_{\max }$, $\alpha\left(f_{\max }\right)$, and $\Delta$, and in the very top, $A_{p}$ index (geomagnetic activity). A vertical line of 8 August indicates the day of earthquake occurrence. This lines refer to the daily values and the full lines indicate the running mean of the daily values over 9 days around the current day. In the plot of $\Delta$, the horizontal line indicates the mean value over one year and dotted horizontal lines indicate the mean plus (and minus) one standard deviation.

has elucidated further characteristics of scaling properties of ULF geomagnetic data for the Guam earthquake.

Our multifractal analysis has yielded a significant insight into the precursory effects of earthquakes. It seems clear that the temporal variations in $w$ and $\Delta$ provide some obvious message for the short-time earthquake forecast. Especially, the non-uniformity factor, $\Delta$ has indicated a significant increase starting about 30 days before and lasting just before the earthquake. This $\Delta$ value is normalized by the denominator $\left(f_{\max }\right)$. The most influential point is the shift of $\alpha_{\min }$ to the left, which indicates that the clustering within the most clustered areas becomes more intense (the local fractal dimension increases within these vicinities). The corresponding increase in $w$ indeed means a transition from homogeneous (random, space filling) to heterogeneous (ordered, complex, clustered) patterns.

We here comment on the 25-30 days precursory warning. The presence of ULF signatures in this period has already been found for a few large earthquakes like Loma Prieta and

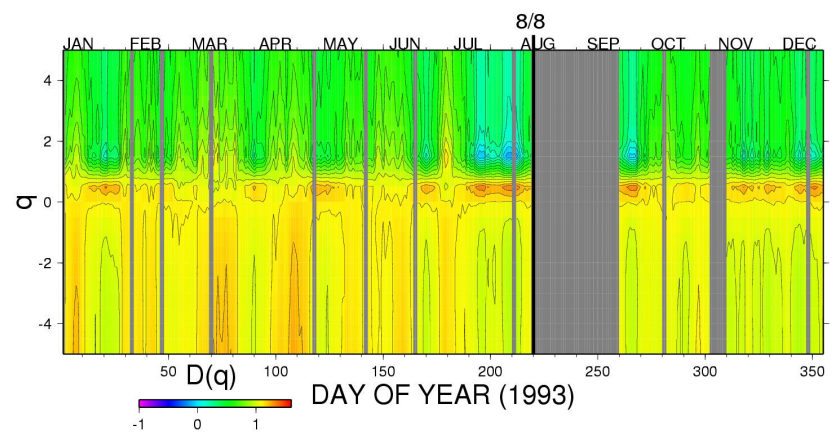

Fig. 5. Temporal evolution of $D q$ (multifractal dimension) during the one year of 1993. The value of $D q$ is indicated in color. Also, the earthquake date of 8 August is given by a vertical line.

some others. This kind of temporal evolution has been attempted to explain by means of the microfracturing model by Molchanov and Hayakawa (1995) based on a space-time model of microfracture progression.

While the multifractality of earthquake patterns has been firmly established by now (Goltz, 1997), there have been very few reports on the use of multifractal spectral change as a precursor to earthquakes. Only a few sources are known to us; Jiang (1993) and Hirabayashi et al. (1992). Jiang (1993) was interested in the change in mutifractal parameters for moderately large earthquakes (long-term variation of the order of a year) and he concludes that the temporal variations in $w$ and $\Delta f$ provide some message for medium-term forecast of earthquakes. Then, the main focus of Hirabayashi (1992)'s paper was on showing that seismicity from three major regions (California, Greece and Eastern Japan) constitutes multifractal measures. But, they analyzed the temporal behavior of the spectrum of generalized dimensions (the $D q$ curve) obtained from the epicenter distributions. The similar work to these has been recently published by Kiyaschenko et al. (2003) in order to find out some medium-term earthquake precursors. The epicenter distributions analyzed by Jiang (1993) and Hirabayashi et al. (1992) are known to be useful for the medium-term prediction, but the short-term earthquake prediction seems to be possible only with the use of electromagnetic phenomena (Hayakawa and Molchanov, 2002). Our mutifractal analysis for the geomagnetic ULF data is found to be of great potential in short-term earthquake prediction. Also, we will take into account the mutifractal changes as obtained in this paper in the improvement of our previous generation mechanism (Molchanov and Hayakawa, 1995).

Acknowledgements. One of the authors (M. H.) is grateful to the Japan Society of Promotion of Science (\#15403012) and the Mitsubishi Foundation for their support.

Edited by: J. Kurths

Reviewed by: two referees 


\section{References}

Bak, P., Tang, C., and Wiesenfeld, K.: Self-organized criticality: An explanation of 1/ $f$ noise, Phys. Rev. Lett., 59, 381-384, 1987.

Bak, P.: How Nature Works (The Science of Self-organized Criticality), Oxford University Press, 201, 1997.

Burlaga, L. F. and Klein, L. W.: Fractal structure of the interplanetary magnetic field, J. Geophys. Res., 91, 347-350, 1986.

Feder, J.: Fractals, Plenum Press, New York, 283, 1988.

Fraser-Smith, A. C., Bernardi, A., McGill, P. R., Ladd, M. E., Helliwell, R. A., and Villard Jr., O. G.: Low-frequency magnetic field measurements near the epicenter of the Ms 7.1 Loma Prieta earthquake, Geophys. Res. Lett., 17, 1465-1468, 1990.

Goltz, C.: Fractal and Chaotic Properties of Earthquakes, SpringerVerlag, Berlin, 178, 1997.

Gotoh, K., Hayakawa, M., and Smirnova, N.: Fractal analysis of the ULF geomagnetic data obtained at Izu peninsula, Japan in relation to the nearly earthquake swarm of June-August 2000, Nat. Haz. Earth Sys. Sci., 3, 229-236, 2003,

\section{SRef-ID: 1684-9981/nhess/2003-3-229.}

Gotoh, K., Smirnova, N., and Hayakawa, M.: Fractal analysis of seismogenic ULF emissions, Special Issue on "Seismo Electromagnetics and Related Phenomena", edited by: Hayakawa, M., Molchanov, O. A., Biagi, P. F., and Vallianatos, V., Phys. Chem. Earth, Parts A/B/C, 29, 4-9, 419-424, 2004.

Gotoh, K., Akinaga, Y., Hayakawa, M., and Hattori, K.: Principal component analysis of ULF geomagnetic data for Izu islands earthuqakes in July 2000, J. Atmos. Electr., 22, 1-12, 2002.

Hayakawa, M. (ed.): Atmospheric and Ionospheric Electromagnetic Phenomena Associated with Earthquakes, Terra Sci. Pub. Comp., Tokyo, 996, 1999.

Hayakawa, M., Molchanov, O. A., and NASDA/UEC Team: Summary report of NASDA's Earthquake Remote Sensing Frontier Project, in: Special Issue on Seismo Electromagnetic and Related Phenomena, edited by: Hayakawa, M., Mochanov, O. A., Biagi, P. F., and Vallianatos, F., Phys. Chem. Earth, 29, 4-9, 617626, 2004.

Hayakawa, M., Itoh, T., and Smirnova, N.: Fractal analysis of ULF geomagnetic data associated with the Guam earthquake on $8 \mathrm{Au}-$ gust 1993, Geophys. Res. Lett., 26, 2797-2800, 1999.

Hayakawa, M., Itoh, T. Hattori, K., and Yumoto, K.: ULF electromagnetic precursors for an earthquake at Biak, Indonesia on 17 February 1996, Geophys. Res. Lett., 27, 1531-1534, 2000.

Hayakawa, M.: NASDA's Earthquake Remote Sensing Frontier Research, Seismo-electromagnetic Phenomena in the Lithosphere, Atmosphere and Ionosphere, Final Report, Univ. of ElectroCommunications, March, 228, 2001.

Hayakawa, M. and Fujinawa, Y.: Electromagnetic Phenomena Related to Earthquake Prediction, Terra Sci. Pub. Comp., Tokyo, Japan, 477, 1994.

Hayakawa, K., Kawate, R., Mochanov, O. A., and Yumoto, K.: Results of ultra-low-frequency magnetic field measurements during the Guam earthquake of 8 August 1993, Geophys. Res. Lett., 23, 241-244, 1996.

Hayakawa, M. and Molchanov, O. A. (Eds): Seismo Electromagnetics: Lithosphere-Atmosphere-Ionosphere Coupling, TERRAPUB, Tokyo, 477, 2002.

Higuchi, T.: Approach to an irregular time on the basis of fractal theory, Physica D, 31, 277-283, 1988.

Hirabayashi, Y., Ito, K., and Yoshi, T.: Multifractal analysis of earthquakes, PAGEOPH, 138, 519, 1992.
Jiang, H. K.: The multifractal local scaling feature of "spatial energy generating" and its seismic precursory information, Int'l Symp. on Fractal and Dynamic Systems in Geoscience, Frankfurt, Book of Abstracts, 1, 12, 1993.

Kantelhardt, J. W., Zschiegner, S. A., Koscielny-Bunde, E., Bunde, A., Havlin, S., and Stanley, H. E.: Multifractal deterended fluctuation analysis of nonstationary time series, Physica A, 316, 8191, 2002.

Kapiris, P. G., Balasis, G. T., Kopanas, J. A., Antonopoulos, G. N., Pertezakis, A. S., and Eftaxias, K. A.: Scaling similarities of multiple fracturing of solid materials, Nonlin. Proc. Geophys., 11, 137-151, 2004,

SRef-ID: 1607-7946/npg/2004-11-137.

Kiyaschenko, D., Smirnova, N., Troyan, V., and Vallianatos, F.: Dynamics of multifractals and correlation characteristics of the spatio-temporal distribution of regional seismicity before the strong earthquakes, Nat. Haz. Earth Sys. Sci., 3, 285-298, 2003, SRef-ID: 1684-9981/nhess/2003-3-285.

Kopytenko, Y. A., Matishvili, T. G., Voronov, P. M., Kopytenko, E. A., and Molchanov, O. A.: Detection of ultra-low-frequency emissions connected with the Spitak earthquake and its aftershock activity, based on geomagnetic pulsations data at Dusheti and Vatdzia observations, Phys. Earth Planet. Inter., 77, 85-95, 1993.

Mandelbort, B.: Multifractal measures, especially for the geophysicist, Pure Appl. Geophys., 131, 5-42, 1989.

Molchanov, O. A., Kopytenko, Y. A., Voronov, P. M., Kopytenko, E. A., Matiashvill, T. G., Fraser-Smith, A. C., and Bernardi, A.: Results of ULF magnetic field measurements near the epicenters of the Spitak $(M s=6.9)$ and Loma Prieta $(M s=7.1)$ earthquakes: Comparative analysis, Geophys. Res. Lett., 19, 14951498, 1992.

Molchanov, O. A. and Hayakawa, M.: Generation of ULF electromagnetic emissions by microfracturing, Geophys. Res. Lett., 22, 3091-3094, 1995.

Smirnova, N. A.: The peculiarities of the ground observed geomagnetic pulsation as the background for detection of the ULF emissions of seismic origin, in: Atmospheric and Ionospheric Electromagnetic Phenomena Associated with Earthquakes, edited by: Hayakawa, M., Terra Sci. Pub. Co., Tokyo, 215-232, 1999.

Smirnova, N., Hayakawa, M., Gotoh, K., and Volobuev, D.: Scaling characteristics of the ULF geomagnetic fields at the Guam seismoactive area and their dynamics in relation to the earthquake, Nat. Haz. Earth Sys. Sci., 1, 119-126, 2001,

SRef-ID: 1684-9981/nhess/2001-1-119.

Troyan, V. N., Smirnova, N. A., Kopytenko, Yu. A., Peterson, Th., and Hayakawa, M.: Development of a complex approach for searching and investigation of electromagnetic precursors of earthquakes: Organization of experiments and analysis procedures, in: Atmospheric and Ionospheric Electromagnetic Phenomena Associated with Earthquakes, edited by: Hayakawa, M., Terra Sci. Pub. Co., Tokyo, 147-170, 1999.

Turcotte, D. L.: Fractals and Chaos in Geology and Geophysics, Cambridge University Press, 221, 1992.

Vallianatos, F. and Tzanis, A.: A model for the generation of precursory electric and magnetic fields associated with the deformation rate of the earthquake focus, in: "Atmospheric and Ionospheric Electromagnetic Phenomena Associated with Earthquakes", edited by: Hayakawa, M., TERRAPUB, Tokyo, 287306, 1999. 